\title{
Interactions between Gemini Surfactants, 12-s-12, and $\beta$-cyclodextrin As Investigated by NMR Diffusometry and Electric Conductometry
}

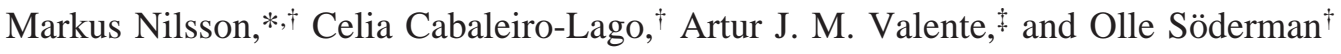 \\ Physical Chemistry 1, Center for Chemistry and Chemical Engineering, Lund University, \\ P. O. Box 124, SE 22100 Lund, Sweden, and Department of Chemistry, University of Coimbra, \\ 3004-535 Coimbra, Portugal
}

Received May 2, 2006. In Final Form: July 13, 2006

\begin{abstract}
The interaction between $\beta$-cyclodextrin (CD) and gemini surfactant of the type alkyl- $\alpha, \omega$-bis(dodecyldimethylammonium bromide) with different spacer lengths of 2, 8, and 10 carbons has been investigated by means of electric conductivity (EC) and proton self-diffusion NMR at $298 \mathrm{~K}$. The formation of a 2:1 (CD:gemini) complex in a two-step mechanism is observed with the first association constant $\left(K_{11}\right)$ higher than the second one $\left(K_{21}\right)$, but both relatively small in comparison with single $C_{12}$-tailed surfactant. The value of the association constants increased with spacer length both for the first and second associated $\mathrm{CD}$, which indicates that the available space on the gemini molecule is important. The magnitudes of the association constant both for the first and second complexation are discussed. The first association constant is small (when compared with the homologous single-chain surfactant) due to hydrophobic interaction between the hydrocarbon tails within the gemini molecule, while the second association constant shows no cooperativity and its magnitude is discussed in terms of steric constrains.
\end{abstract}

\section{Introduction}

Gemini surfactants are made up of two amphiphilic moieties connected at the level of the headgroups or very close to the headgroups by a spacer group. ${ }^{1-3}$ These surfactants are characterized by a much lower $\mathrm{cmc}$ (critical micelle concentration), and by a higher efficiency to decrease the surface tension of water, than those for the corresponding monomeric surfactants; ${ }^{4}$ aqueous solutions of some cationic surfactant dimers with short spacers can have a very high viscosity at a relatively low surfactant concentration and/or display viscoelasticity and shear induced viscoelasticity. ${ }^{5}$ The properties of gemini surfactants are greatly influenced by the length of the spacer group, ${ }^{6}$ hydrophobic chain length, and dissymmetry. ${ }^{7}$ They also have a high surface activity, better foaming properties, and the ability to make organic compounds soluble in water, ${ }^{8}$ which can be applied to different fields such as wastewater treatment ${ }^{9}$ and DNA compaction. ${ }^{10}$

The use of these surfactants in multiple areas can be increased with the addition of cyclodextrins. Cyclodextrins (CDs) are cyclic oligosaccharides ${ }^{11}$ in the shape of a truncated cone composed, in the more common forms, of six to eight $(\alpha-1,4)$-linked $\alpha$-Dglucopyranose units with internal cavities ranging from 5 to 8 $\AA$ ( $\alpha$-, $\beta$-, and $\gamma$-cyclodextrin). Due to this structure CDs readily form inclusion complexes through noncovalent interactions with molecular guests. Surfactants are particular guest compounds. The beneficial modification of guest molecular properties after the formation of an inclusion compound leads to a large number

\footnotetext{
$\doteqdot$ Lund University.

$\doteqdot$ University of Coimbra.

(1) Menger, F. M.; Littau, C. A. J. Am. Chem. Soc. 1991, 113, 1451-1452.

(2) Menger, F. M.; Littau, C. A. J. Am. Chem. Soc. 1993, 115, 10083-10090.

(3) Zana, R.; Benrraou, M.; Rueff, R. Langmuir 1991, 7, 1075-1075.

(4) Zana, R. J. Colloid Interface Sci. 2002, 248, 203-220.

(5) Schmitt, V.; Schosseler, F.; Lequeux, F. Europhys. Lett. 1995, 30, 31-36.

(6) Danino, D.; Talmon, Y.; Zana, R. Langmuir 1995, 11, 1448-1456.

(7) Oda, R.; Huc, I.; Candau, S. J. Chem. Commun. (Cambridge) 1997, 21 2105-2106.

(8) Menger, F. M.; Keiper, J. S. Angew. Chem., Int. Ed. 2000, 39, 1907-1920. (9) Benalla, H.; Meziani, M. J.; Zajac, J. Colloids Surf., A 2004, 238, 99-108. (10) Karlsson, L.; Eijk, M. C. P. v.; Söderman., O. J. Colloid Interface Sci. 2002, 252, 290-296.
}

(11) Uekama, K.; Hirayama, F.; Irie, T. Chem. Rev. 1998, 98, 2045-2076. of applications related to chemical synthesis and catalysis, ${ }^{12}$ pharmaceutical chemistry, ${ }^{13}$ and analytical chemistry. ${ }^{14}$ Interactions between cyclodextrins and surfactants have been analyzed by a number of authors and techniques, such as fluorescence, ${ }^{15}$ nuclear magnetic resonance, ${ }^{16,17}$ conductometry, ${ }^{18}$ surface tension, ${ }^{19}$ and calorimetry, ${ }^{20}$ etc. However, only a few papers ${ }^{12,21,22}$ are related to interactions between gemini surfactants and cyclodextrins.

The aim of this paper is to investigate the interaction between gemini surfactants, with different spacer lengths (12-s-12), and $\beta$-cyclodextrin using ${ }^{1} \mathrm{H}$ NMR self-diffusion and electrical conductivity measurements. With NMR diffusometry direct information about the state of the components compromising the system, that is, surfactant, $\beta$-cyclodextrin, and complexes of surfactant and $\beta$-cyclodextrin, is obtained. The conductivity data will give us overall and therefore indirect information about the alterations in the ionic structure of the surfactant solution upon $\beta$-cyclodextrin addition. The calculation of the complexation ratio and binding constants will help us to elucidate the interaction mechanism between surfactant and $\beta$-cyclodextrin.

\section{Experimental Procedure}

2.1. Materials and Solutions. $\beta$-Cyclodextrin (CD) was purchased from Aldrich with a water content of $13.1 \mathrm{wt} \%$ (molar

(12) Jiang, B. Y.; Zang, R. R.; Xie, J. Q.; J. Du; Meng, X. G.; Zeng, X. C. J. Dispersion Sci. Technol. 2005, 26, 105-110.

(13) Stella, V. J.; Rajewski, A. Pharm. Rev. 1997, 14, 556-567.

(14) Li, S. P., W. C. Chem. Rev. 1992, 92, 2045-2076.

(15) Wintgens, V.; Amiel, C. J. Photochem. Photobiol., A 2004, 168, 217226.

(16) Valente, A. J. M.; Nilsson, M.; Söderman, O. J. Colloid Interface Sci. 2005, 281, 218-224.

(17) Karlson, L.; Malmborg, C.; Thuresson, K.; Söderman, O. Colloids Surf. A 2003, 228, 171-179.

(18) Rafati, A. A.; Bagheri, A.; Iloukhani, H.; Zarinehzad, M. J. Mol. Liq 2005, 116, 37-41.

(19) Galant, C.; Wintgens, W.; Amiel, C.; Auvray, L. Macromolecules 2005, $38,5243-5253$

(20) Abdala, A. A.; Tonelli, A. E.; Khan, S. A. Macromolecules 2003, 36, $7833-7841$

(21) Alami, E.; Abrahmsén-Alami, S.; Eastoe, J.; Grillo, I.; Heenan, R. K. J. Colloid Interface Sci. 2002, 255, 403-409.

(22) Abrahmsén-Alami, S.; Alami, E.; Eastoe, J.; Cosgrove, T. J. Colloid Interface Sci. 2002, 246, 191-202. 
Table 1. Stock Solutions Prepared for Different Gemini/CD Samples

\begin{tabular}{ccc}
\hline gemini & {$[$ gemini] $/ \mathrm{mM}($ set I) } & {$[\mathrm{CD}] / \mathrm{mM}($ set II $)$} \\
\hline $12-2-12$ & 0.83 & 2.77 \\
$12-8-12$ & 0.82 & 2.77 \\
$12-10-12$ & 0.63 & 2.77
\end{tabular}

Table 2. Values of $\mathrm{cmc}$ (in the Absence of CD, from Conductivity Data (This Study) and from the Literature ${ }^{36}$ and Observed and Estimated cac (in the Presence of CD, $2.77 \mathrm{mM}$ )

\begin{tabular}{ccccc}
\hline gemini & $\mathrm{cmc}^{a} / \mathrm{mM}$ & $\mathrm{cmc}^{b} / \mathrm{mM}$ & $\mathrm{cac} / \mathrm{mM}$ & $\begin{array}{c}\text { estd cac } c / \mathrm{mM} \\
(\mathrm{cac}=([\mathrm{cmc}+0.5[\mathrm{CD}]))\end{array}$ \\
\hline $12-2-12$ & $0.89 \pm 0.02$ & 0.83 & 2.0 & 2.21 \\
$12-8-12$ & $0.80 \pm 0.01$ & 0.82 & 1.9 & 2.20 \\
$12-10-12$ & $0.64 \pm 0.07$ & 0.63 & 1.6 & 1.98
\end{tabular}

${ }^{a}$ Conductivity results from this study. ${ }^{b}$ Values taken from ref 36. ${ }^{c}$ Estimated surfactant concentrations above which micelles are present, assuming a 2:1 CD:gemini complex of infinite association constant.

ratio of $m \mathrm{H}_{2} \mathrm{O}: n C D$ is 17:2) as determined from thermal analysis. The concentrations of CD solutions were corrected by assuming such water content (hexahydrated compound). Gemini surfactants ethyl- $\alpha, \omega$-bis(dodecyldimethylammonium bromide) (12-2-12), octyl- $\alpha, \omega$-bis(dodecyldimethylammonium bromide) (12-8-12), and decyl- $\alpha, \omega$-bis(dodecyldimethylammonium bromide) (1210-12) were kindly provided by Prof. Jason Keiper (Emory University, Atlanta, GA) and were used without further purification. The $n$-dodecyltrimethylammonium bromide was purchased from Tokyo Kasei and also used without further purification.

The NMR samples were prepared using $\mathrm{D}_{2} \mathrm{O}(99.8 \%)$, supplied by Dr. Glaser AG (Basel, Switzerland), as solvent. Two sets of stock solution were prepared: the first set (set I) is when the surfactant (12-s-12) concentration is kept constant and the concentration of CD changes, and the second set (set II) is when the concentration of $\mathrm{CD}$ is constant varying the concentration of (12-s-12). Table 1 shows the different stock solutions for set I and set II for all three different geminis. It should be noted that according to conductivity determination of cmc (see Table 2) the 12-8-12 stock solution concentration is slightly above the cmc.

The samples were prepared by weighing the proper amount of solute (CD or surfactant) and the corresponding stock solution. The tubes were sealed with tightened Teflon caps and equilibrated.

The solutions used in the conductometric technique were prepared by using Millipore-Q water $\left(\kappa=(0.7-0.9) \times 10^{-4} \mathrm{~S}\right.$ $\left.\mathrm{m}^{-1}\right)$, and all solutions were freshly prepared just before each experiment.

2.2. Methods. Pulsed field gradient (PFG) ${ }^{1} \mathrm{H}$ NMR experiments were performed on a $200 \mathrm{MHz}$ Bruker DMX spectrometer equipped with a Bruker DIFF-25 gradient probe driven by a Bruker BAFPA-40 unit. All the experiments were carried out at controlled probe temperatures $\left(25.0 \pm 0.5^{\circ} \mathrm{C}\right)$ in $5 \mathrm{~mm} \mathrm{NMR}$ test tubes.

The self-diffusion coefficients of surfactants $D_{\mathrm{S}}$ and cyclodextrin $D_{\mathrm{CD}}$ were obtained using pulse-gradient spin-echo proton NMR $\left({ }^{1} \mathrm{H}\right.$ PGSE) following the recommendations in previous works. ${ }^{23,24}$ The gradient strengths $(G)$ were changed from 4.82 to $9.63 \mathrm{~T} \mathrm{~m}^{-1}$, and the duration time of the gradient pulse $(\delta)$ was kept constant at $0.5 \mathrm{~ms}$. The diffusion time (the time between leading edges of the field gradient pulses; $\Delta$ ) was typically $20-$ $100 \mathrm{~ms}$.

For molecules undergoing unhindered random motion and for a single species the attenuation of the signal intensity is

(23) Söderman, O.; Stilbs, S. Prog. Nucl. Magn. Reson. Spectrosc. 1994, 26, 445-482.

(24) Stilbs, P. Prog. Nucl. Magn. Reson. Spectrosc. 1987, 19, 1-45. given by

$$
I=I_{0} \exp \left[-\gamma^{2} G^{2} \delta^{2}\left(\Delta-\frac{\delta}{3}\right) D\right]
$$

In eq $1, I$ denotes the observed intensity, $I_{0}$ is the intensity in the absence of gradient pulses, $\gamma$ is the magnetogyric ratio, and the rest of the quantities are defined above.

The experimental data were analyzed using a nonlinear leastsquares fitting procedure, IKFIT (an in-house-developed software, based on the Matlab package (version 6.1)) to extract the $D$ from an exponential decay. The cyclodextrin and surfactants selfdiffusion coefficients were calculated using the IKFIT analysis, with at least 10 experimental data points and with an echo decay intensity variation higher than 1 order of magnitude. ${ }^{16}$ The uncertainty in the fit of eq 1 to the data is in general much smaller than $1 \%$, and it was obtained through a Monte Carlo analysis following the procedure suggested in previous works. ${ }^{25}$

Solution electrical resistances were measured with a WayneKerr model 4265 Automatic LCR meter at $1 \mathrm{kHz}$. A Shedlovskytype conductance cell, with a cell constant of approximately $0.8465 \mathrm{~cm}^{-1}$, was used in the measurements. ${ }^{26}$ Measurements were made at $25.00 \pm 0.01^{\circ} \mathrm{C}$ in a Grant thermostat bath. Solutions were always used within $12 \mathrm{~h}$ after preparation. In a typical experiment a surfactant solution was placed in the conductivity cell; then, aliquots of the CD solution were added in a stepwise manner using a micropipet. The conductance of the solution was measured ca. $10 \mathrm{~min}$ after each addition. The $\mathrm{cmc}$ of aqueous solutions of $12-s-12$ is obtained from the empirical data of specific conductance $(\kappa)$ as a function of surfactant concentration by calculating the zero of the third derivative of $\kappa=f(c)$, and the values are reported in Table 2.

\section{Experimental Results}

Proton self-diffusion NMR and electrical conductivity are used in this study to investigate the host-guest association between $\mathrm{CD}$ and gemini surfactant. The outlay of Experimental Results will begin with a discussion concerning the stoichiometries based on the NMR data; there information from both species is obtained. The second part of the outline will rely on data from the electrical conductivity, which data set consists of much more data points than the NMR due to the fact that the host (CD) have been titrated into the sample cell, and thereby will be used for determination of the value of the binding constants.

3.1. NMR Diffusometry. The association process between gemini surfactants $(\mathrm{S})$ and the $\beta$-cyclodextrin has been studied by varying the concentration of $\mathrm{CD}$ for a constant concentration of surfactant just below its cmc value (this series was designated as set I above). Figure 1 shows the variation of the self-diffusion coefficients for the CD:12-2-12 system. Similar trends have been observed for the other gemini surfactants.

When analyzing the trends of the self-diffusion in Figure 1, one notes several important features. The self-diffusion of the surfactant goes down upon the addition of cyclodextrin and reaches a plateau at approximately a concentration of $3.5 \mathrm{mM}$ $\mathrm{CD}$, while the self-diffusion of the $\mathrm{CD}$ decreases until a concentration of $1.6 \mathrm{mM} \mathrm{CD}$ and after that increases in the rest of the concentration interval approaching the value for the selfdiffusion coefficient of $\mathrm{CD}$ in $\mathrm{D}_{2} \mathrm{O}$ (cf. the dashed line in Figure 1). The minimum in the CD self-diffusion coefficients curve cannot be explained if the complex has a 1:1 stoichiometry.

(25) Alper, J. S.; Gelb, R. I. J. Phys. Chem. 1990, 94, 4747-4751.

(26) Ribeiro, A. C. F.; Valente, A. J. M.; Lobo, V. M. M.; Azevedo, E. F. G.; Amado, A. M.; da Costa, A. M. A.; Ramos, M. L.; Burrows, H. D. J. Mol. Struct. 2004, 703, 93-101. 


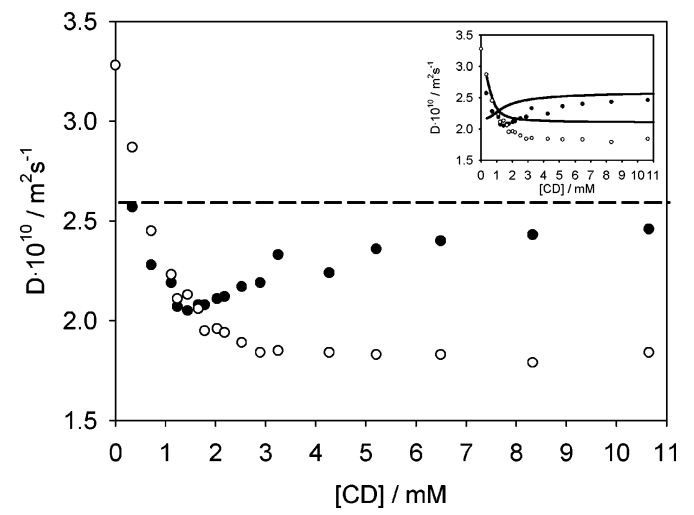

Figure 1. Self-diffusion coefficients, $D$, for $\mathrm{CD}(\bullet)$ and gemini $(12-2-12)(O)$, varying the concentration of $C D$ for $[12-2-12]=0.83$ $\mathrm{mM}$. The insert shows the same data and the prediction of a $1: 1$ (CD:gemini) model, where the binding constant is $12000 \mathrm{M}^{-1}$ has been used. The dashed line corresponds to the self-diffusion of free $\mathrm{CD}$ in $\mathrm{D}_{2} \mathrm{O}$.

Table 3. Self-Diffusion Coefficients for the Species in CD:Gemini Mixtures As Obtained by ${ }^{1} \mathrm{H}$ NMR Measurements

\begin{tabular}{lccc}
\hline & $12-2-12$ & $12-8-12$ & $12-10-12$ \\
\hline$D_{\mathrm{S}} \times 10^{10} /\left(\mathrm{m}^{2} \mathrm{~s}^{-1}\right)$ & 3.28 & 3.00 & 2.80 \\
$D_{\mathrm{CD}} \times 10^{10} /\left(\mathrm{m}^{2} \mathrm{~s}^{-1}\right)$ & 2.64 & 2.64 & 2.64 \\
$D_{11} \times 10^{10} /\left(\mathrm{m}^{2} \mathrm{~s}^{-1}\right)$ & 2.10 & 2.00 & 1.90 \\
$D_{21} \times 10^{10} /\left(\mathrm{m}^{2} \mathrm{~s}^{-1}\right)$ & 1.80 & 1.75 & 1.65
\end{tabular}

Under the conditions used in Figure 1 and considering the formation of a 1:1 complex which has a lower self-diffusion coefficient than the unassociated $\mathrm{CD}$, the $\mathrm{CD}$ self-diffusion coefficients should increase smoothly and then possibly reach a constant value, depending on the value of the association constant (as an insert we show in Figure 1 the predictions if the only complex formed was 1:1, using parameters from Table 3 , and a value of $\left.K=12000 \mathrm{M}^{-1}\right) \cdot{ }^{27}$ In Figure 1, the addition of a small amount of $\mathrm{CD}$ reduces the self-diffusion coefficient of the $\mathrm{CD}$ due to the formation of complexes of different (higher) stoichiometry. Further addition of $\mathrm{CD}$ causes a progressive increase of the self-diffusion coefficients as the fraction of unassociated $\mathrm{CD}$ increases. For the surfactant, the self-diffusion coefficient decreases as the fraction of associated surfactant increases. The plateau reached indicates that essentially all surfactant is associated, and the plateau value defines the selfdiffusion coefficient for the complex with the highest stoichiometry. Similar observations have been noticed for the hexadecyltrimethylammonium bromide, $\mathrm{C}_{16} \mathrm{TAB}$, and $\mathrm{CD}$ system ${ }^{27}$ and also here the observed trends were attributed to and explained in terms of an association with a stoichiometry higher than 1:1.

On the assumption that complexes of stoichiometries 1:1 and 2:1 CD:S are formed, the self-diffusion coefficients of all species present can be estimated and are tabulated in Table 3 . The values for unassociated $\mathrm{S}$ and $\mathrm{CD}$ are obtained in separate measurements. The self-diffusion coefficient of the 2:1 complex is taken as the value at the plateau in Figure 1. Finally, to obtain the self-diffusion of the 1:1 complex, the sizes of the 1:1 and 2:1 complexes are considered and eq 2 is used to estimate the self-diffusion of the 1:1 complex.

$$
\frac{D_{21}}{D_{11}}=\left(\frac{V_{11}}{V_{21}}\right)^{1 / 3}
$$

(27) Cabaleiro-Lago, C.; Nilsson, M.; Söderman, O. Langmuir 2005, 21, $11637-11644$.

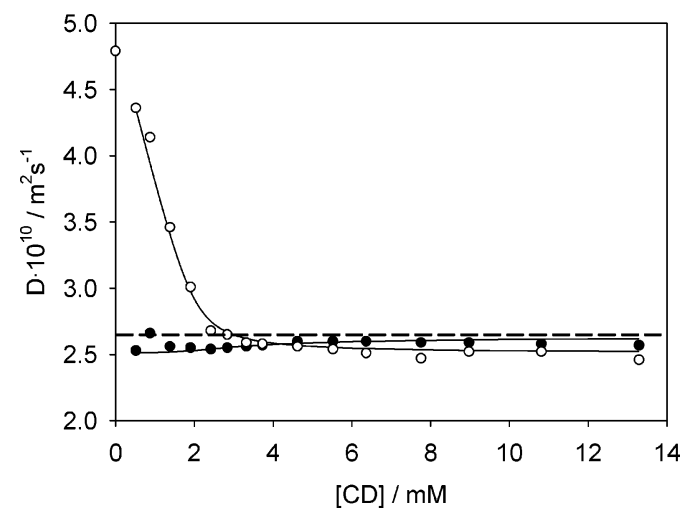

Figure 2. Self-diffusion coefficients, $D$, for $\mathrm{CD}(\bullet)$ and DoTAB $(\mathrm{O})$, varying the concentration of $\mathrm{CD}$ for $[\mathrm{DoTAB}]=2.0 \mathrm{mM} \cdot{ }^{27} \mathrm{The}$ dashed line corresponds to the self-diffusion of free $\mathrm{CD}$ in $\mathrm{D}_{2} \mathrm{O}$.

$V_{11}$ and $V_{21}$ are the volumes of the 1:1 and 2:1 complex, respectively. For the calculations the volume of $\mathrm{CD}$ is taken as $1325 \AA^{3}$ and is estimated from density values of glucose. ${ }^{28}$ For the geminis, the following group volumes have been used: $V_{\mathrm{CH}_{2}}$ $=27 \AA^{3}, V_{\mathrm{CH}_{3}}=55 \AA^{3}$, and $V_{\mathrm{N}-\left(\mathrm{CH}_{3}\right)_{2}}=75 \AA$.

The association between $\mathrm{CD}$ and the analogue single-chain version of the gemini surfactant, dodecyltrimethylammonium bromide, DoTAB, has been studied earlier, ${ }^{27}$ and data are included here as a reference in Figure 2. The self-diffusion of CD is essentially constant in the whole region. The self-diffusion data of $\mathrm{CD}$ and DoTAB have been fitted to a model assuming association into 1:1 complexes, and the association constant obtained was $K_{11}=18600 \pm 4000 \mathrm{M}^{-1} .{ }^{27}$ The CD self-diffusion remains almost constant on account of the fact that the 1:1 complex has approximately the same diffusion coefficient as unassociated $\mathrm{CD}$. The different trend observed between gemini and DoTAB can be attributed to the formation of complexes with different stoichiometries as discussed above.

To further investigate the complexation stoichiometry, NMR self-diffusion experiments on samples with constant concentration of $\mathrm{CD}$ (see Table 1) and varying concentrations of gemini were performed for all three geminis, and data are presented in Figure 3. The $\mathrm{cmc}$ of the geminis in the absence of $\mathrm{CD}$ are known from the literature but also measured here with conductometry as a control and are tabulated in Table 2. The concentration of surfactant above which micelles form (called here critical aggregation constant, cac) in the presence of CD conveys information of the complexation stoichiometry. For the case of a 1:1 complexation, cac should be equal to $\mathrm{cac}=\mathrm{cmc}+[\mathrm{CD}]$ or lower if the association process is weak. If the complex between $\mathrm{S}$ and CD is only 2:1, complete association leads to $\mathrm{cac}=(\mathrm{cmc}$ $+0.5[\mathrm{CD}])$, but if we consider weaker associations cac $<$ ( $\mathrm{cmc}$ $+0.5[\mathrm{CD}]) .{ }^{29}$ The discontinuity in Figure 3 signals the formation of micelles, and the cac for the geminis from the data (see Table 2) suggests that complexes of stoichiometries $1: 1$ and 2:1 are formed. In principle, it would be possible to analyze the data in Figure 1 with a model based on the presence of $1: 1$ and 2:1 complexes and to estimate values of the binding constants. However, this requires access to a large number of high-precision data points, which is not practical to obtain from NMR measurements on account of the rather low inherent sensitivity of NMR (please note that the concentrations are rather low, on account of the rather low cmc values).

Conductometry offers a way to obtain large data sets with a high degree of precision, and we shall use this technique to

(28) Nilsson, F.; Söderman, O.; Johansson, I. Langmuir 1996, 12, 902-908

(29) Garcia-Rio, L.; Leis, J. R.; Mejuto, J. C.; Perez-Juste, J. J. Phys. Chem. B 1997, 101, 7383-7389. 


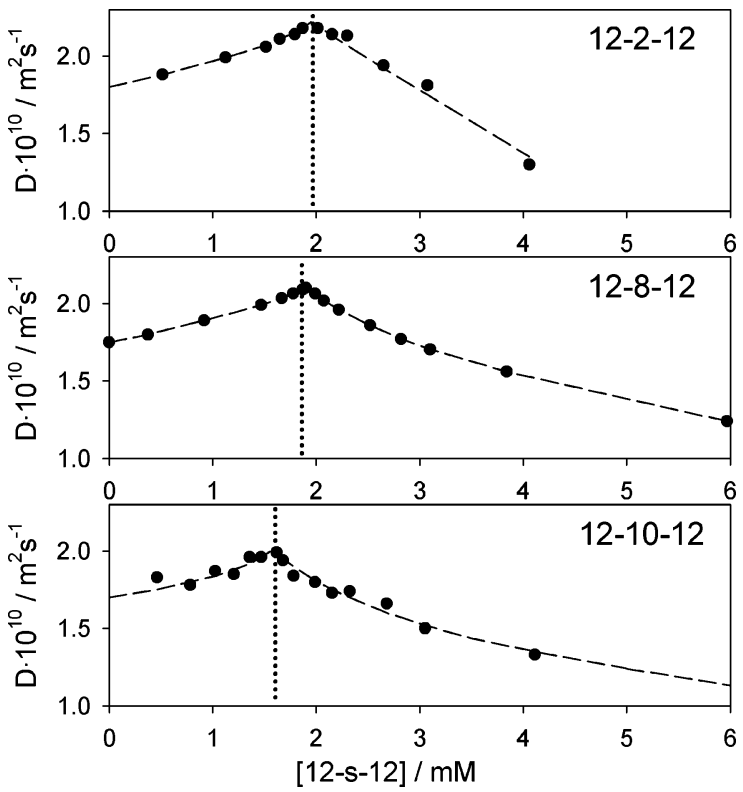

Figure 3. Self-diffusion coefficients for gemini (a) 12-2-12, (b) 12-8-12, and (c) 12-10-12 vs the concentration of gemini surfactant $[\mathrm{CD}]=2.77 \mathrm{mM}$. Dotted lines show the cac values (see Table 2 ). Dashed lines are guides for the eye.

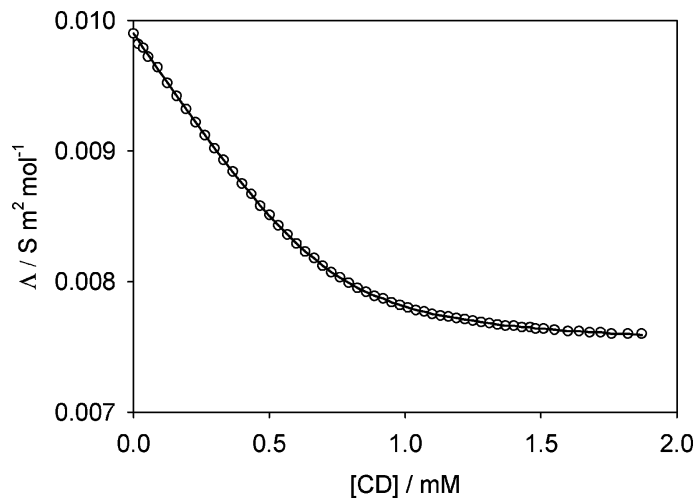

Figure 4. Dependence of the molar conductivity of the DoTAB $(0.732 \mathrm{mM})$ on the $\beta$-cyclodextrin concentration. The solid line represents molar conductivities predicted by eq 11 , assuming $1: 1$ $(\mathrm{CD}: \mathrm{S})$ association, using the following parameters: $\Lambda_{\mathrm{S}}=101.2 \times$ $10^{-4} \mathrm{~S} \mathrm{~m}^{2} \mathrm{~mol}^{-1}, \Lambda_{\mathrm{CD}-\mathrm{S}}=76.78 \times 10^{-4} \mathrm{~S} \mathrm{~m}^{2} \mathrm{~mol}^{-1}$, and $K_{11}=$ $17300 \pm 1500 \mathrm{M}^{-1}$.

obtain values of the association constants. In this process, we will use the information from the NMR experiment that complexes of $1: 1$ and 2:1 stoichiometries are formed.

3.2. Electrical Conductivity. We have measured the effect of adding $\beta$-cyclodextrin on the specific conductance of aqueous solutions of DoTAB and gemini surfactants, 12-2-12, 12-8-12, and $12-10-12$, respectively, in the premicellar region, at 25.00 ${ }^{\circ} \mathrm{C}$. The molar conductivity $(\Lambda)$ was calculated using

$$
\Lambda=\left(\kappa-\kappa_{0}\right) /(1000 C)
$$

where $\kappa$ and $\kappa_{0}$ are specific conductance of the solutions and water, respectively, and $C$ is the surfactant concentration in $\mathrm{mol} /$ $\mathrm{dm}^{3}$. Before turning to the results from the gemini surfactant, we consider the single-chain surfactant DoTAB. The molar conductivities for one surfactant concentration and varying $C D$ concentrations are given in Figure 4. In general, the molar conductivity decreases as CD is added, the reason being that as the surfactant ions become complexed by $\mathrm{CD}$, they become less effective as charge carrier on account of the decreased diffusion

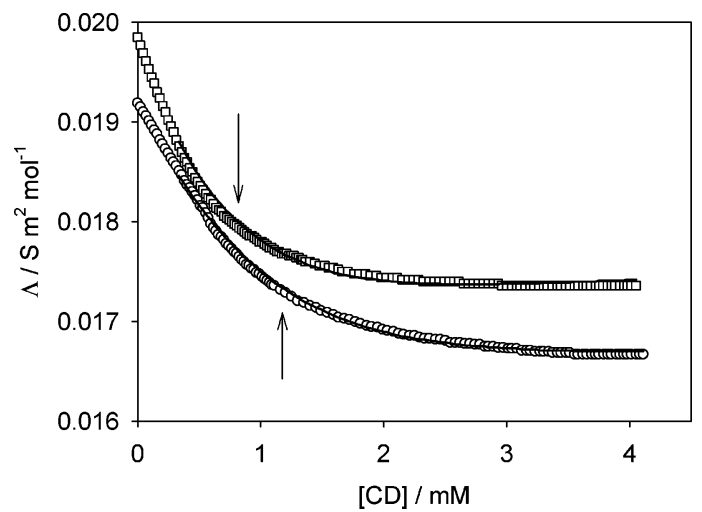

Figure 5. Dependence of the molar conductivity of the 12-2-12 on the $\beta$-cyclodextrin concentration, at constant concentrations of $12-$ 2-12: ( $\square$ ) $0.498 \mathrm{mM}$; (O) $0.758 \mathrm{mM}$. Solid lines represent molar conductivities predicted by eq 11 , assuming $2: 1(\mathrm{CD}: \mathrm{S})$ association, using the parameters shown in Table 5. The arrows in the figure indicate the location where the initial approximate linear decrease of the molar conductivity data with CD concentration ends (see discussion in connection to Table 4).

Table 4. Values of Stoichiometric Ratios [CD]/[12-s-12] Assuming an Infinitely Large Equilibrium Constant

\begin{tabular}{llll}
\hline & \multicolumn{2}{c}{ conductivity } & self-diffusion \\
\hline$[12-2-12] / \mathrm{mM}$ & 0.498 & 0.758 & 0.83 \\
{$[\mathrm{CD}] /[12-2-12]$} & 1.6 & 1.6 & 1.5 \\
{$[12-8-12] / \mathrm{mM}$} & 0.494 & 0.750 & 0.82 \\
{$[\mathrm{CD}] /[12-8-12]$} & 1.8 & 1.9 & 1.8 \\
{$[12-10-12] / \mathrm{mM}$} & 0.380 & 0.491 & 0.63 \\
{$[\mathrm{CD}] /[12-10-12]$} & 1.8 & 2.1 & 2.5
\end{tabular}

coefficient of the complex, as compared to the surfactant alone (as borne out by the diffusion data in Figure 2). When analyzing the DoTAB data assuming that a 1:1 complex is formed (and using equations analogues to the ones presented below), ${ }^{26}$ the association constant obtained was $K_{11}=17300 \pm 1500 \mathrm{M}^{-1}$. The association constant evaluated from the NMR data in Figure 2 was $18600 \pm 4000 \mathrm{M}^{-1} .{ }^{27}$ The values for $K_{11}$ are thus similar for both methods, which means that we can use the qualitative behavior from NMR and quantitative results from the conductivity measurements. Finally, we note that the experimental curve for DoTAB is well-predicted by the $1: 1 \operatorname{model}^{27}$ (cf. Figure 4).

We now turn to the conductivity data for the gemini surfactants, presented in Figure 5. At low CD concentrations, an approximately linear decrease of the molar conductivity with increasing $C D$ concentration is evident. Upon further addition of $\mathrm{CD}$ a rather smoothly changing slope of the curve appears until a plateau is reached at high concentrations of $\mathrm{CD}$. The arrows in Figure 5 indicate approximately where the linear dependence of the CD concentration ends. The intersection of the initial decrease of $\Lambda$ with [CD] and the constant value of $\Lambda$ have been used to give a rough estimate of the CD:gemini stoichiometry. A similar approach can be used for the NMR self-diffusion data. Table 4 summarizes the stoichiometric ratios obtained for the different systems. Although the approach only gives a rough stoichiometric ratio of $\mathrm{CD}: \mathrm{S}$ association, it should be noticed that there is a reasonable agreement between the ratio values calculated by self-diffusion and by conductivity. They suggest the formation of a complex with an association 2:1 (CD:S) in a two-step mechanism.

\section{Modeling the Association Process}

On the basis of the discussion above, our analysis rests on the assumption that the interaction between gemini surfactants and $\beta$-cyclodextrin leads to a 2:1 complexation. If the complexation 
Table 5. Association Constants for the Inclusion Complexes CD:12-s-12 at $25.00{ }^{\circ} \mathrm{C}$, Assuming a 2:1 Complexation in Two Steps

\begin{tabular}{cccccr}
\hline $\mathrm{S}$ & {$[\mathrm{S}]_{t} / \mathrm{mM}$} & $\Lambda_{\mathrm{S}} \times 10^{3} /\left(\mathrm{S} \mathrm{m}^{2} \mathrm{~mol}^{-1}\right)$ & $\Lambda_{\mathrm{CD}-\mathrm{S}} \times 10^{3} /\left(\mathrm{S} \mathrm{m}^{2} \mathrm{~mol}^{-1}\right)$ & $\Lambda_{\mathrm{CD}_{2}-\mathrm{S} \times 10^{3} /\left(\mathrm{S} \mathrm{m}^{2} \mathrm{~mol}^{-1}\right)} K_{11} / \mathrm{M}^{-1}$ & $K_{21} / \mathrm{M}^{-1}$ \\
\hline $12-2-12$ & 0.498 & $19.78 \pm 0.01$ & $16.15 \pm 0.12$ & $17.88 \pm 0.19$ & $1970 \pm 150$ \\
& 0.758 & $19.25 \pm 0.01$ & $15.83 \pm 0.11$ & $16.94 \pm 0.01$ & \\
$12-8-12$ & 0.494 & $19.95 \pm 0.01$ & $17.12 \pm 0.19$ & $17.70 \pm 0.06$ & $3150 \pm 530$ \\
& 0.750 & $18.96 \pm 0.01$ & $16.94 \pm 0.13$ & $16.87 \pm 0.02$ & $1340 \pm 270$ \\
$12-10-12$ & 0.388 & $19.97 \pm 0.01$ & $17.05 \pm 0.34$ & $17.76 \pm 0.08$ & $3130 \pm 790$ \\
& 0.491 & $19.20 \pm 0.01$ & $17.12 \pm 0.25$ & $16.98 \pm 0.04$ & $2120 \pm 430$
\end{tabular}

occurs in a two-step fashion, two equilibrium equations can be written as

$$
\begin{gathered}
\mathrm{CD}+\mathrm{S} \stackrel{K_{11}}{\rightleftarrows} \mathrm{CD}-\mathrm{S} \\
\mathrm{CD}-\mathrm{S}+\mathrm{CD} \stackrel{K_{21}}{\rightleftarrows} \mathrm{CD}_{2}-\mathrm{S}
\end{gathered}
$$

The stability of the inclusion complexes, $\mathrm{CD}-\mathrm{S}$ and $\mathrm{CD}_{2}-\mathrm{S}$, can be described in terms of the association constants, $K_{11}$ and $K_{21}$ :

$$
\begin{gathered}
K_{11}=[\mathrm{CD}-\mathrm{S}] /\left([\mathrm{CD}]_{\mathrm{f}}[\mathrm{S}]_{\mathrm{f}}\right) \\
K_{21}=\left[\mathrm{CD}_{2}-\mathrm{S}\right] /\left([\mathrm{CD}]_{\mathrm{f}}\left[\mathrm{CD}{ }_{-\mathrm{S}}\right]\right)
\end{gathered}
$$

where $[C D]_{\mathrm{f}}$ and $[S]_{\mathrm{f}}$ are the concentration of uncomplexed species in the system. Under these conditions, the observed specific conductance, $\kappa$, can be expressed as

$$
\kappa=\kappa_{\mathrm{S}}+\kappa_{\mathrm{CD}-\mathrm{S}}+\kappa_{\mathrm{CD}_{2}-\mathrm{S}}
$$

where $\kappa_{\mathrm{S}}, \kappa_{\mathrm{CD}-\mathrm{S}}$, and $\kappa_{\mathrm{CD}_{2}-\mathrm{S}}$ are specific conductances of the surfactant and complexes.

Conservation of mass gives

$$
\begin{gathered}
{[\mathrm{S}]_{\mathrm{f}}=[\mathrm{S}]_{\mathrm{T}}-[\mathrm{CD}-\mathrm{S}]-\left[\mathrm{CD}_{2}-\mathrm{S}\right]} \\
{[\mathrm{CD}]_{\mathrm{f}}=[\mathrm{CD}]_{\mathrm{T}}-[\mathrm{CD}-\mathrm{S}]-2\left[\mathrm{CD}_{2}-\mathrm{S}\right]}
\end{gathered}
$$

where $[S]_{\mathrm{T}}$ and $[C D]_{\mathrm{T}}$ are the total concentration of surfactant and cyclodextrin, respectively.

Combination of eqs 6-10 gives, after some algebra, the following expression for the observed molar conductivity:

$$
\Lambda=\frac{\Lambda_{\mathrm{S}}+\Lambda_{\mathrm{CD}-\mathrm{S}} K_{11}[\mathrm{CD}]_{\mathrm{f}}+\Lambda_{\mathrm{CD}_{2}}-\mathrm{S} K_{11} K_{21}[\mathrm{CD}]_{\mathrm{f}}^{2}}{1+K_{11}[\mathrm{CD}]_{\mathrm{f}}+K_{11} K_{21}[\mathrm{CD}]_{\mathrm{f}}^{2}}
$$

The free cyclodextrin concentration $[C D]_{\mathrm{f}}$ is given by

$$
\begin{aligned}
{[\mathrm{CD}]_{\mathrm{f}}^{3}+} & \left(\frac{1}{K_{21}}-[\mathrm{CD}]_{\mathrm{T}}+2[\mathrm{~S}]_{T}\right)[\mathrm{CD}]_{\mathrm{f}}^{2}+ \\
& \left(\frac{1}{K_{11} K_{21}}+\frac{[\mathrm{S}]_{\mathrm{T}}}{K_{21}}-\frac{[\mathrm{CD}]_{\mathrm{T}}}{K_{21}}\right)[\mathrm{CD}]_{\mathrm{f}}-\frac{[\mathrm{CD}]_{\mathrm{T}}}{K_{11} K_{21}}=0
\end{aligned}
$$

Equation 12 can be solved to obtain the concentration of free $\mathrm{CD},[\mathrm{CD}]_{\mathrm{f}}$, using standard procedures. The association constants, $K_{11}$ and $K_{21}$, are obtained from a least-squares fit of eqs 11 and 12 to the molar conductivities. Least-squares fits were performed using in-house written software based on the Matlab package (version 6.1). To increase the accuracy in the obtained parameters, global fits have been performed using experimental data from two different surfactant concentrations. The calculated association constants and other fitting parameters are given in Table 5. It should be noted that in the fitting procedure two separate values of $\Lambda_{\mathrm{S}}$ and $\Lambda_{\mathrm{CD}-\mathrm{S}}$ have been used for each total surfactant concentration. Differences in the calculated values of $\Lambda_{S}$ and $\Lambda_{\mathrm{CD}-\mathrm{S}}$ are small, which also reflects small variations of equilibrium surfactant and complex concentrations. Molar conductivities decrease with an increase of $[\mathrm{S}]_{\mathrm{T}}$, suggesting the reliability of the model even if other factors, such as the variation of the dissociation degree, possible ion-pair formation, and variation of properties of ions and solvent, etc., that can affect the mobility of the species in solution, ${ }^{30}$ are neglected.

\section{Discussion}

5.1. Complexation Stoichiometries. All the geminis studied here contain at least 30 carbons (in the case of 12-2-12) and a maximum of 38 (in the case of 12-10-12). From that point of view the complexation stoichiometry should be at least 2:1 $\left(K_{21}\right)$, since a surfactant with 16 carbons in the tail is known to form a 2:1 complexes with cyclodextrin. ${ }^{27}$ A special feature of the gemini surfactants is the possibility of complexation on the spacer, i.e., a CD molecule would bind between the two charged headgroups. That was shown by Cabaleiro-Lago et al., ${ }^{31}$ who studied the complexation with a bola surfactant having 12 carbons between the two charged groups (in the nomenclature used here, the bola surfactant would be designated 1-12-1), although we note that the maximum spacer length used here is 10. From the studies of the association of a bolaform surfactant with $\alpha-C D$, it can be inferred that the headgroups exert some repulsive force on the $\mathrm{CD}$ ring to stay outside the cavity. ${ }^{32}$ That explains why short bolas with less that six methylene groups, do not form complexes with $\alpha-C D$. The stability of the complexes increases as the length of the alkyl chain increases, and complexes between bolaform surfactants with alkyl chains longer than eight methylene groups with $\alpha$-cyclodextrin have been found. ${ }^{32-35}$ Taking into account that the depth of the CD cavity is the same for $\alpha$ - and $\beta$-cyclodextrin, it is reasonable expect that a molecule of $\mathrm{CD}$ associates to the alkyl chain spacer between the headgroups for 12-8-12 and 12-10-12, although with a weak association constant. We see no indication for this in our data, but cannot exclude the possibility that a weak association complex is formed at the spacer. An interesting question related to this is the lifetime of the complex. Presumably, the barrier for the formation of such a complex is considerable (data to support this for the case of $\alpha-C D$ are given in ref 34 ). We see no indication of slow exchange from the NMR diffusion data (the NMR intensities were always exponentially decaying; cf. eq 1), indicating that the exchange

(30) Robinson, R. A.; Stokes, R. H. Electrolyte Solutions; 2nd ed.; Dover: New York, 2002

(31) Cabaleiro-Lago, C.; Nilsson, M.; Valente, A. J. M.; Bonini, M.; Soderman, O. NMR Diffusometry and Conductometry Study of the Host-Guest Association between Cyclodextrin and Dodecane 1,12-Bis(trimethylammonium Bromide). J. Colloid Interface Sci. 2006, 300, 782-787.

(32) Wenz, G.; Han, B.-H.; Müller, A. Chem. Rev. 2006, 106, 782-817.

(33) Jin, V. X.; Macartney, D. H.; Buncel, E. J. Inclusion Phenom. Mol. Recognit Chem. 2005, 53, 197-203.

(34) Avram, L.; Cohen, Y. J. Org. Chem. 2002, 67, 2639-2644.

(35) Lyon, A. P.; Banton, N. J.; Macartney, D. H. Can. J. Chem. 1998, 76, $843-850$.

(36) Zana, R. J. Colloid Interface Sci. 2002, 248, 203-220. 
is more rapid than the characteristic time of the NMR experiment, which is on the order of $50 \mathrm{~ms}$.

That a mixture of (at least) 1:1 and 2:1 complexes is present at higher concentration of $\mathrm{CD}$ can be inferred from the fact that the $1: 1$ fitting fails to reproduce the curve (both methods) and the $2: 1$ model reproduces the experimental curve well in the case of the electrical conductivity measurements. Furthermore, a comparison of the self-diffusion trends between the gemini 122-12 (Figure 1) and with a DoTAB surfactant (Figure 2) clearly shows differences in the self-diffusion behavior as $\mathrm{CD}$ is added to the surfactant solutions.

5.2. Magnitudes of the Binding Constants and the Influence of the Spacer Length. Two features are apparent in the data presented in Table 5. First, for the three geminis studied, the association constant $K_{11}$ is higher than $K_{21}$, but $K_{21}$ approaches $K_{11}$ when the spacer length increases (see Table 5). Thus the binding of CD to the surfactant is anti-cooperative. Second, the magnitude of $K_{11}$ is $5-10$ times smaller than the value for the single-chain C12 surfactant (cf. above).

In what follows we will discuss these observations, starting with the latter feature. As a starting point we note that there is a possibility of hydrophobic interactions between the two chains in the gemini. As a consequence, the free energy gain to form the complex between the first gemini chain and CD is less than what would be the case for a single-chain surfactant of the same chain length. From the ratio of the association constants for the gemini and the corresponding single-chain surfactant, it is straightforward to estimate the change in free energy between the two cases, it amounts to roughly $30 \%$ (calculated with $K_{11}$ $=2 \times 10^{4}$ for the single chain and $K_{11}=2 \times 10^{3}$ for the gemini). Since the free energy change to first approximation is proportional to the hydrocarbon area exposed to water, this would imply that the difference in area exposed before and after association is on the order of $30 \%$ between the single-chain and gemini surfactants, which does not sound unreasonable. We note that the difference between the gemini and the single chain is expected to decrease as the spacer in the gemini is made longer; this effect is born out by the data in Table 5 .

With regard to the cooperativity, one we perhaps intuitively would expect a cooperative binding of the second CD bind to the remaining chain of the gemini. However, this is not the case. The effect is most pronounced for 12-2-12. Now, this effect is probably due to steric effects. Once one CD molecule has associated with the gemini, the available space for the second $\mathrm{CD}$ to associate with the free chain is limited. We present in Figure 6 space filling pictures of 1:1 inclusion complexes for 12-2-12 and 12-8-12, illustrating the rather crowded situation once one $\mathrm{CD}$ is complexed to the gemini. There is a further effect of electrostatic origin. The gemini has two charges. If both chains are complexed to $\mathrm{CD}$ molecules, the charges will be surrounded by an environment rich in methyl groups, which is unfavorable from an electrostatic point of view. Finally, we note that the value of $K_{21}$ increases and approaches the value of $K_{11}$ as the spacer increases in length. This is the expected result as the gemini becomes more flexible and the two tails approach a situation where they are independent of each other.

Finally, as mentioned above, there is an interesting question with regard to the spacer between the two quaternary nitrogens. In a recent article, ${ }^{31}$ we have shown that $\mathrm{CD}$ forms a 1:1 inclusion complex with a bola surfactant of 12 carbons. The longest spacer used here is 10 carbons. It is conceivable that the 12:10:12 gemini could from a 3:1 inclusion complex with one CD complexed on the level of the spacer, forming a rotaxane-like structure, and further CDs complex to the gemini hydrocarbon chains. As
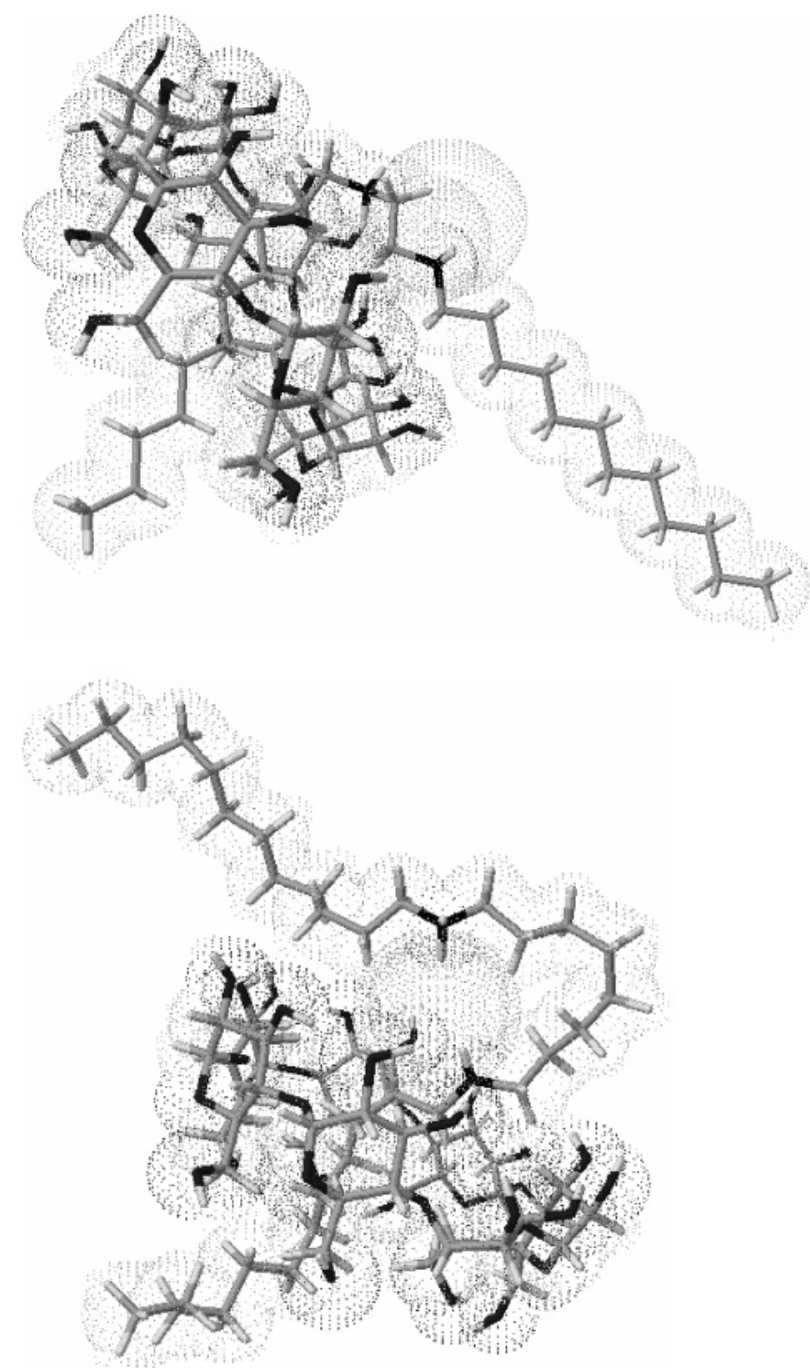

Figure 6. Schematic representation of the structure of a 1:1 inclusion complex between CD and (top) 12-2-12 and (bottom) 12-8-12. The figures were generated using the CambridgeSoft package (CS ChemBats3D Pro).

argued above, if such a complex would be formed, the association constant for the $\mathrm{CD}$ complexed to the spacer would be rather low, on account of the steric and electrostatic arguments given below. To shed further light on the structure of the complexes, we are investigating the complexes using two-dimensional NMR techniques. The results of these studies will be presented elsewhere.

\section{Conclusions}

The model used to evaluate the stoichiometry and association constants reproduces the experimental behavior well for the conductometric techniques used in this study. The highest stoichiometry was shown to be $2: 1(\mathrm{CD}: \mathrm{S})$ for the three gemini spacer lengths investigated. The values of the association constants $K_{11}$ and $K_{21}$ increased with increasing spacer length, although the former was equal for the spacers with 8 and 10 methylene groups. The binding constants for $K_{11}$ were compared with the analogue DoTAB (as measured with conductivity and NMR diffusometry) and were found to be 1 order of magnitude smaller. We suggest an explanation for the low binding constants for $K_{11}$ and also for $K_{21}$, based on the hydrophobic interaction between the hydrocarbon tails on the gemini and (for the case of $K_{11}$ ) and steric constraints and electrostatic effects for the comparatively low value of $K_{21}$. For 12-10-12, the binding constants were the 
same, indicating that the molecule was flexible enough and that the two tails start to be independent of each other.

Acknowledgment. We thank Professor Victor Lobo for valuable discussion on conductivity analysis. Financial support from VR, FCT, Fundação Calouste Gulbenkian is gratefully acknowledged. C.C.-L. thanks the Ministerio de Educación y Ciencia for a FPU fellowship.

LA061220E 